\title{
The Relationships Between Quantitative MR Parameters in Hippocampus in Healthy Subjects and Patients With Temporal Lobe Epilepsy
}

\author{
D. WAGNEROVÁ ${ }^{1}$, V. HERYNEK ${ }^{1}$, M. DEZORTOVÁ ${ }^{1}$, P. MARUSIČ $^{2}$, P. KRŠEK $^{3}$, \\ J. ZÁMEČNÍK ${ }^{4}$, F. JÍRŮ ${ }^{1}$, A. ŠKOCH ${ }^{1}$, M. HÁJEK ${ }^{1}$
}

${ }^{1}$ Department of Diagnostic and Interventional Radiology, Institute for Clinical and Experimental Medicine, Prague, Czech Republic, ${ }^{2}$ Department of Neurology, Charles University in Prague, Second Faculty of Medicine, Motol University Hospital, Prague, Czech Republic, ${ }^{3}$ Department of Pediatric Neurology, Charles University in Prague, Second Faculty of Medicine, Motol University Hospital, Prague, Czech Republic, ${ }^{4}$ Department of Pathology and Molecular Medicine, Charles University in Prague, Second Faculty of Medicine, Motol University Hospital, Prague, Czech Republic

Received June 11, 2014

Accepted November 21, 2014

On-line December 22, 2014

\section{Summary}

We introduce a new magnetic resonance (MR) method based on a pixel-by-pixel image processing to examine relationships between metabolic and structural processes in the pathologic hippocampus. The method was tested for lateralization of the epileptogenic zone in patients with temporal lobe epilepsy (TLE). Twenty patients with drug-resistant TLE and fifteen healthy controls were examined at $3 \mathrm{~T}$. The measurement protocol contained T2-weighted MR images, spectroscopic imaging, diffusion tensor imaging and $\mathrm{T} 2$ relaxometry. Correlations between quantitative MR parameters were calculated on a pixelby-pixel basis using the CORIMA program which enables automated pixel identification in the normal tissue according to control data. All MR parameters changed in the anteroposterior direction in the hippocampus and correlation patterns and their slopes differed between patients and controls. Combinations of T2 relaxation times with metabolite values represent the best biomarkers of the epileptogenic zone. Correlations with mean diffusivity did not provide sufficiently accurate results due to diffusion image distortions. Quantitative MR analysis noninvasively provides a detailed description of hippocampal pathology and may represent complementary tool to the standard clinical protocol. However, the automated processing should be carefully monitored in order to avoid possible errors caused by MR artifacts.

\section{Key words}

Temporal lobe epilepsy • 1H-MR spectroscopy • MR diffusometry - T2 relaxometry $\bullet$ Correlations

\section{Corresponding author}

D. Wagnerová, Institute for Clinical and Experimental Medicine, Vídeňská 1958/9, 14021 Prague, Czech Republic. Fax: +420241728224. E-mail: diwa@medicon.cz

\section{Introduction}

Magnetic resonance (MR) techniques enable to study different biochemical and biophysical parameters of the brain tissue in vivo. MR images based on T1 and $\mathrm{T} 2$ relaxation times (T1, T2 resp.), diffusion maps or metabolite concentration maps are broadly used in clinical practice for localizing the pathology. Nevertheless, standard MRI provides only morphological information and the routinely used visual paradigm often fails in non-lesional diseases. Temporal lobe epilepsy (TLE) represents a typical epileptic syndrome where 
more detailed data analysis provides additional information about the disease and can help in better comprehension of pathogenic mechanisms.

TLE is potentially surgically-remediable focal epilepsy. Symptomatic TLE is etiologically divided into the syndrome of mesial temporal lobe epilepsy (MTLE) associated with hippocampal sclerosis (HS), lesional TLE caused by different structural lesions, and cryptogenic TLE (Engel 1996). HS is characterized by hippocampal and extrahippocampal atrophy with neuronal loss and astrogliosis in the hippocampus (HC) and in the surrounding regions (Margerison and Corsellis 1966, Bonilha et al. 2010).

Sclerotic HC exhibits significantly increased mean diffusivity (MD) ipsilaterally to the epileptogenic focus (Duzel et al. 2004, Concha et al. 2009) or bilaterally (Keller and Roberts 2008, Knake et al. 2009, Bonilha et al. 2010). An increase of T2 ipsilaterally (Jack 1996) was attributed to the increased number of glial cells (Pereira et al. 2006). Higher $\mathrm{T} 2$ relaxation times in the anterior rather than posterior $\mathrm{HC}$ were also reported (von Oertzen et al. 2002, Bartlett et al. 2007).

Proton magnetic resonance spectroscopy (1H-MRS) assessing concentrations of selected metabolites reveals neuronal dysfunction characterized by $\mathrm{N}$-acetylaspartate $+\mathrm{N}$-acetylaspartylglutamate (NAA) decrease, reactive astrogliosis accompanied by creatine (Cr) increase or a dysplastic cortical lesion characterized by choline-containing compounds (Cho) increase. Abnormal $\mathrm{NAA} /(\mathrm{Cho}+\mathrm{Cr})$ ratios were found in the ipsilateral and sometimes in the contralateral temporal lobe in the TLE patients (Kuzniecky et al. 2001, Hajek et al. 2008).

Several studies concluded that pathologic extent and lateralization of the epileptogenic zone (EZ) in ambiguous cases may be better resolved by a combination of different diagnostic methods and correlations between different parameters. Negative correlations were found between $\mathrm{NAA} /(\mathrm{Cho}+\mathrm{Cr})$ and apparent diffusion coefficient (ADC) or the T2 values in the sclerotic HC (Namer et al. 1999, Kantarci et al. 2002, Pereira et al. 2006). Significant correlations between ADC and T2 were observed in the temporal lobe in the patients as well as in the control subjects (Duzel et al. 2004).

The aim of this work was to verify the ability of a new MR method based on a pixel-by-pixel image processing to study the relationship between individual biochemical or biophysical parameters and to use their combination for an examination of the hippocampi in healthy controls and in patients with TLE. The findings based on correlations between $1 \mathrm{H}-\mathrm{MRS}$, T2 relaxometry and diffusometry were compared with EZ lateralization using standard pre-surgical evaluations and histopathological data available after epilepsy surgery.

\section{Methods}

\section{Subjects}

Twenty TLE patients (mean age 29.6 \pm 12.4 years) evaluated before planned resective surgery for intractable epilepsy in Motol Epilepsy Center were involved in the study. Demographic and clinical data are summarised in Table 1. Fifteen patients had abnormal radiological findings in the hippocampus classified as lesional epilepsy while the remaining five patients were regarded non-lesional. The surgical side was determined according to the electro-clinical correlation and the results of all pre-operative investigations. Fifteen patients underwent temporal lobe resection and, accordingly, their histopathological findings were available (Table 1).

Fifteen healthy subjects (mean age 24.0 \pm 2.2 years), carefully interviewed to exclude any diseases affecting the obtained data, were included in this study as controls.

All subjects provided their informed consent approved by the local ethical committee. Clinical protocols are certified according to the ISO 9001:2008 norm.

\section{MR measurements}

The patients underwent a standard clinical MRI at $1.5 \mathrm{~T}$ used for radiological evaluation. Consequently, the patients underwent a supplementary examination on a $3 \mathrm{~T}$ tomograph (Siemens Medical Systems, Erlangen, Germany) equipped with a transmit-receive head coil aimed on DTI, spectroscopic imaging (SI) and T2 relaxometry. The images were positioned in parallel to the long hippocampal axis and special attention was paid to the symmetric positioning over both HC.

1H-MR spectroscopy: The SI data were obtained using a 2D Point Resolved Spectroscopy (PRESS-CSI) sequence with echo time (TE)/repetition time (TR)/number of acquisitions (NA) $=135 \mathrm{~ms} / 1510 \mathrm{~ms} / 4$, field of view (FOV) $160 \times 160 \times 15 \mathrm{~mm}^{3}$, a $16 \times 16$ phase encoding matrix, nominal voxel volume $1.5 \mathrm{ml}$, with and without water suppression.

T2 relaxometry: A modified 32-echo Carr- 
Purcell-Meiboom-Gill (CPMG) sequence was used with the following parameters: $\mathrm{TR}=3000 \mathrm{~ms}$, echospacing $\Delta \mathrm{TE}=13.2 \mathrm{~ms}, \mathrm{NA}=1$, slice thickness $(\mathrm{ST})=5 \mathrm{~mm}$. FOV was adjusted according to the patient's head size with a constant in-plane pixel size $0.78 \times 0.78 \mathrm{~mm}^{2}$.

Diffusion tensor imaging: DTI data were acquired using an echo planar imaging spin echo diffusion tensor pulse sequence (TR/TE/NA $=7100 \mathrm{~ms} /$ $98 \mathrm{~ms} / 3,20$ directions, 44 slices, $\mathrm{ST}=2.5 \mathrm{~mm}$ without a slice gap, FOV adjusted according to the patient's head size with a constant in-plane pixel size $2.0 \times 2.0 \mathrm{~mm}^{2}, \mathrm{~b}=0$; $1000 \mathrm{~s} / \mathrm{mm}^{2}$ ).

The entire protocol was performed in twelve patients, $\mathrm{T} 2$ relaxometry was omitted in four patients, and DTI also in four patients. In two patients, the MRS data were available only from one $\mathrm{HC}$ due to technical reasons.

\section{Data processing}

SI data were analyzed using a jSIPRO program (Jiru et al. 2013) with LCModel (Provencher 1993). The data processing included Hamming k-space filtering and the zero filling to a $32 \times 32$ matrix size. A water signal was used as an internal calibration for the calculation of the metabolic concentration in the laboratory units. The data were corrected neither for the relaxation times nor for the content of cerebrospinal fluid as it was unnecessary for the proposed method and the exact absolute concentrations were not the stated aim of the study.

DTI data were processed with FSL 4.1.5 (http://www.fmrib.ox.ac.uk/fsl/). Eddy current, motion artifact corrections and averaging of the individual data acquisitions was performed. Brain extraction with a fractional intensity threshold equal 0.3 was undertaken. Mean diffusivity was calculated in each voxel from the brain-extracted data using the FSL DTIfit tool.

T2 relaxation maps were calculated using the ViDi program (Herynek et al. 2012). Image preprocessing included the image matrix reduction (to in-plane resolution $1.56 \times 1.56 \mathrm{~mm}^{2}$ to adapt it to the spectroscopic resolution and to increase the signal-tonoise ratio (SNR)), and exclusion of points with a low SNR prior to the fitting.

The conventional MR images were visually assessed by experienced neuroradiologists and epileptologists.

\section{Correlation analysis}

Correlations between metabolic images and diffusion or $\mathrm{T} 2$ relaxation maps were calculated using the CORIMA program (Wagnerova et al. 2009). The correlations were based on a pixel-by-pixel evaluation of two different MR methods which allows studying mutual dependences of MR parameters. The software enables a user to select a region of interest (ROI) in one map (metabolite, relaxation, or diffusion), automatically find the corresponding region in another map and consequently create the correlation plot. Each point inside the plot then corresponds to one pixel inside the selected ROI. Hippocampal ROIs were selected manually according to MRI. Both hippocampi were evaluated separately in each subject. The metabolic maps as well as maps of metabolic ratios were used. Only voxels with an error (Cramer-Rao bound) of the calculated metabolic concentrations lower than $15 \%$ were included in the analysis (Jiru et al. 2006). Areas in which the relaxation times were not calculated due to low SNR or a failure of the fitting algorithm were excluded from the T2 correlations.

The program facilitates the automatic identification of pixels in the normal tissue according to the control data, highlights them on corresponding MR maps and then shows regions with parameter abnormalities and pathology extent. The minimum (MIN) and maximum (MAX) values for each MR parameter in the hippocampus were assessed as a Mean - standard deviation (SD) of the control data set, and Mean + SD, respectively. High MD values in the vicinity of the brain stem were excluded from the control data set. MIN and MAX values define the control data interval (highlighted in correlation plots by shading and bordered by blue lines) used for the semiautomatic evaluation of patients' data for each image (Wagnerova et al. 2009). The program automatically interpreted each pixel falling inside this interval as representing a healthy tissue, and pixels with metabolic, MD or T2 values outside this interval as pathologic (Table 1, columns MRS-MD and MRS-T2 denoting correlations between metabolites and MD, T2 resp.). Significant changes in both HC were interpreted as bilateral pathology. Bilateral changes with significant asymmetry in at least one MR parameter were interpreted as bilateral pathology with one side predominance.

A linear fit was used for the correlation analysis. Mann-Whitney U-test was used for comparison of correlation slopes between different patient groups and controls. The probability level $\mathrm{p}<0.05$ was considered as a statistically significant difference. 
Table 1. Demographic, electro-clinical, radiological, correlation and histopathological data of all patients involved in the study.

\begin{tabular}{|c|c|c|c|c|c|c|}
\hline $\begin{array}{l}\text { Patient } \\
\text { No., sex }\end{array}$ & $\begin{array}{c}\text { Age/epilepsy } \\
\text { duration } \\
\text { (years) }\end{array}$ & $\begin{array}{c}\text { Electro-clinical } \\
\text { diagnosis }\end{array}$ & MRI finding & $\begin{array}{c}\text { MRS-MD } \\
\text { lateralization }\end{array}$ & $\begin{array}{c}\text { MRS-T2 } \\
\text { lateralization }\end{array}$ & $\begin{array}{c}\text { Histopathological } \\
\text { finding }\end{array}$ \\
\hline $1, \mathrm{~m}$ & $32 / 14$ & TLE sin & normal & $\mathrm{HC} \sin$ & $\mathrm{HC} \sin$ & no surgery \\
\hline $2, \mathrm{f}$ & $34 / 19$ & TLE $\sin$ & normal & $\mathrm{HC} \sin$ & NA & no surgery \\
\hline $3, \mathrm{f}$ & $35 / 21$ & TLE $\sin$ & normal & HC bilat (sin) & NA & no surgery \\
\hline $4, \mathrm{~m}$ & $20 / 6$ & TLE $\sin$ & normal & NA & HC bilat (sin) & normal \\
\hline $5, \mathrm{f}$ & $19 / 12$ & MTLE $\mathrm{dx}$ & normal $^{\#}$ & $\mathrm{HC} d x$ & $\mathrm{HC} \mathrm{dx}$ & FCD 1B \\
\hline $6, \mathrm{~m}$ & $40 / 14$ & MTLE sin & $\begin{array}{l}\mathrm{T} 2 \mathrm{~W} \text { increased } \\
\text { signal in left } \mathrm{T} \text { pole }\end{array}$ & HC bilat (sin) & HC bilat (sin) & $\begin{array}{l}\text { HC non-specific } \\
\text { gliosis, FCD } 2 \mathrm{~A}\end{array}$ \\
\hline $7, \mathrm{f}$ & $41 / 23$ & MTLE sin & $\begin{array}{l}\text { T2W increased } \\
\text { signal in left PHG }\end{array}$ & NA & normal $\mathrm{HCdx}$ * & $\begin{array}{l}\text { HC non-specific } \\
\text { gliosis, FCD 1B }\end{array}$ \\
\hline $8, \mathrm{f}$ & $18 / 8$ & MTLE dx & $\operatorname{HS} d x$ & $\mathrm{HC}$ bilat $(\sin )$ & HC bilat (dx) & isolated HS \\
\hline $9, \mathrm{~m}$ & $20 / 7$ & MTLE dx & HS dx & $\mathrm{HC} \mathrm{dx}$ & $\mathrm{HC} \mathrm{dx}$ & isolated HS \\
\hline $10, \mathrm{f}$ & $20 / 15$ & MTLE sin & $\mathrm{HS} \sin$ & HC bilat (sin) & $\mathrm{HC} \sin$ & isolated HS \\
\hline $11, \mathrm{f}$ & $18 / 14$ & MTLE sin & $\begin{array}{l}\text { HS sin, T2W } \\
\text { increased signal and } \\
\text { atrophy in T pole }\end{array}$ & $\mathrm{HC} \sin$ & HC bilat (sin) & HS, FCD 1A \\
\hline $12, \mathrm{~m}$ & $41 / 9$ & MTLE dx & $\begin{array}{l}\text { HS dx } \\
\text { HS sin,T2W }\end{array}$ & $\mathrm{HC}$ bilat & $\mathrm{HC}$ bilat $(\mathrm{dx})$ & HS, FCD 1A \\
\hline $13, \mathrm{f}$ & $15 / 11$ & MTLE sin & $\begin{array}{l}\text { increased signal and } \\
\text { atrophy in T pole }\end{array}$ & $\mathrm{HC} \sin$ & $\mathrm{HC} \sin$ & HS, FCD 1B \\
\hline $14, \mathrm{~m}$ & $32 / 3$ & MTLE sin & HS sin & HC bilat (sin) & NA & HS, FCD 1B \\
\hline $15, \mathrm{~m}$ & $18 / 6$ & MTLE sin & $\begin{array}{l}\mathrm{HS} \text { sin, T2W } \\
\text { increased signal and } \\
\text { atrophy in T pole }\end{array}$ & $\mathrm{HC} \sin$ & $\mathrm{HC} \sin$ & $\begin{array}{l}\text { HS, post- } \\
\text { inflammation } \\
\text { gliosis }\end{array}$ \\
\hline $16, \mathrm{~m}$ & $57 / 10$ & MTLE dx & HS dx & HC bilat (dx) & $\mathrm{HC}$ bilat & $\begin{array}{l}\text { HS, post- } \\
\text { inflammation } \\
\text { gliosis }\end{array}$ \\
\hline $17, \mathrm{~m}$ & $53 / 37$ & MTLE dx & HS dx & $\mathrm{HC}$ bilat $(\mathrm{dx})$ & $\mathrm{HC}$ bilat (dx) & no surgery \\
\hline $18, \mathrm{f}$ & $34 / 34$ & TLE $d x$ & HS dx & NA & $\mathrm{HCdx}$ & no surgery \\
\hline $19, \mathrm{~m}$ & $27 / 20$ & MTLE dx & $\begin{array}{l}\text { T2W increased } \\
\text { signal in right } \\
\text { amygdala, PHG and } \\
\text { HC }\end{array}$ & NA & HC bilat (dx) & $\begin{array}{l}\text { complex } \\
\text { malformation of } \\
\text { HC, FCD 2B }\end{array}$ \\
\hline $20, \mathrm{f}$ & $18 / 1$ & TLE $\mathrm{dx}$ & $\begin{array}{l}\text { right basal posterior } \\
\text { temporal tumour }\end{array}$ & $\mathrm{HC} \mathrm{dx} *$ & NA & $\begin{array}{l}\text { ganglioglioma, } \\
\text { FCD 1A }\end{array}$ \\
\hline
\end{tabular}

MRS-MD and MRS-T2 correlations were evaluated in the hippocampi only. MRS - magnetic resonance spectroscopy, MD - mean diffusivity, $\mathrm{T} 2-\mathrm{T} 2$ relaxation time, $\mathrm{m}$ - male, $\mathrm{f}-$ female, $\mathrm{dx}$ - right, sin - left, $\mathrm{T}$ - temporal lobe, PHG - parahippocampal gyrus, bilat $(\mathrm{dx})$ - bilateral pathology with the right side predominance, bilat (sin) - bilateral pathology with the left side predominance, (M)TLE (mesial) temporal lobe epilepsy, T2W - T2 weighted images, HS - hippocampal sclerosis, HC - hippocampus, WM - white matter, MCD - malformation of cortical development, FCD - focal cortical dysplasia, NA - not available, * - diagnosed with developmental anterobasal temporal encephalocele, * - data from $\mathrm{HC}$ sin not available. 
Table 2. Correlation slope, type and correlation coefficient between selected parameters in controls and patients' abnormal hippocampi.

\begin{tabular}{|c|c|c|c|c|c|c|}
\hline & Controls & $\begin{array}{c}\text { Non-lesional } \\
\text { HC }\end{array}$ & $\begin{array}{c}\text { Lesional } \\
\text { HC }\end{array}$ & Controls & $\begin{array}{c}\text { Non-lesional } \\
\text { HC }\end{array}$ & $\begin{array}{c}\text { Lesional } \\
\text { HC }\end{array}$ \\
\hline \multirow{2}{*}{ Correlation } & Correlation & Correlation & Correlation & Correlation & Correlation & Correlation \\
\hline & Slope A & Slope A & Slope A & Type (r) & Type (r) & Type (r) \\
\hline MD-T2 & $23000 \pm 14600$ & $21200 \pm 5200$ & $18300 \pm 1100$ & $\mathrm{P}(0.38 \pm 0.16)$ & $\mathrm{P}(0.47 \pm 0.11)$ & $\mathrm{P}(0.61 \pm 0.16)$ \\
\hline Cr/NAA-T2 & $0.006 \pm 0.004$ & $0.013 \pm 0.003^{+}$ & $0.011 \pm 0.004^{\#}$ & $\mathrm{P}(0.59 \pm 0.19)$ & $\mathrm{P}(0.59 \pm 0.12)$ & $\mathrm{P}(0.76 \pm 0.09)$ \\
\hline Cr/NAA-MD & $280 \pm 220$ & $620 \pm 500$ & $180 \pm 110$ & $\mathrm{P}(0.32 \pm 0.17)$ & $\mathrm{P}(0.41 \pm 0.19)$ & $\mathrm{P}(0.49 \pm 0.21)$ \\
\hline Cho/NAA-T2 & $0.005 \pm 0.002$ & $0.005 \pm 0.002$ & $0.003 \pm 0.001$ & $P(0.68 \pm 0.13)$ & $\mathrm{P}(0.64 \pm 0.13)$ & $P(0.76 \pm 0.07)$ \\
\hline Cho/NAA-MD & $240 \pm 160$ & $230 \pm 260$ & $80 \pm 40^{\#}$ & $\mathrm{P}(0.46 \pm 0.17)$ & $\mathrm{P}(0.45 \pm 0.22)$ & $\mathrm{P}(0.47 \pm 0.20)$ \\
\hline Cho/Cr-T2 & $0.004 \pm 0.001$ & $0.0018 \pm 0.0009$ & $0.0008 \pm 0.0008^{\#}$ & $\mathrm{P}(0.64 \pm 0.17)$ & $\mathrm{P}(0.63 \pm 0.12)$ & $\varnothing(0.37 \pm 0.38)$ \\
\hline Cho/Cr-MD & $170 \pm 120$ & $30 \pm 110$ & $9 \pm 30^{\#}$ & $P(0.44 \pm 0.25)$ & $\varnothing(0.28 \pm 0.40)$ & $\varnothing(0.21 \pm 0.37)$ \\
\hline Cho-T2 & $0.010 \pm 0.008$ & $0.013 \pm 0.004^{*}$ & $0.005 \pm 0.002^{*}$ & $\mathrm{P}(0.49 \pm 0.17)$ & $\mathrm{P}(0.68 \pm 0.13)$ & $\mathrm{P}(0.74 \pm 0.05)$ \\
\hline Cho-MD & $420 \pm 370$ & $410 \pm 410$ & $110 \pm 80$ & $\mathrm{P}(0.37 \pm 0.27)$ & $\mathrm{P}(0.37 \pm 0.21)$ & $\mathrm{P}(0.47 \pm 0.22)$ \\
\hline $\mathrm{Cr}-\mathrm{T} 2$ & $-0.01 \pm 0.02$ & $0.020 \pm 0.006^{*+}$ & $0.008 \pm 0.006^{* \#}$ & $\varnothing(-0.05 \pm 0.43)$ & $\mathrm{P}(0.59 \pm 0.15)$ & $\mathrm{P}(0.59 \pm 0.23)$ \\
\hline Cr-MD & $-530 \pm 1160$ & $960 \pm 450^{*}$ & $230 \pm 200^{*}$ & $\varnothing(-0.03 \pm 0.38)$ & $\mathrm{P}(0.39 \pm 0.09)$ & $\mathrm{P}(0.39 \pm 0.23)$ \\
\hline NAA-T2 & $-0.04 \pm 0.02$ & $-0.022 \pm 0.018$ & $-0.021 \pm 0.008$ & $\mathrm{~N}(-0.62 \pm 0.13)$ & $\mathrm{N}(-0.38 \pm 0.20)$ & $\mathrm{N}(-0.74 \pm 0.06)$ \\
\hline NAA-MD & $-2180 \pm 1840$ & $-1360 \pm 2090$ & $-490 \pm 330^{\#}$ & $\mathrm{~N}(-0.30 \pm 0.18)$ & $\varnothing(-0.26 \pm 0.35)$ & $\mathrm{N}(-0.54 \pm 0.12)$ \\
\hline
\end{tabular}

Mean value and its standard deviation over each group was calculated for correlation slope $A$ and correlation coefficient $r$. Equation of linear regression $y=A . x+B$ was used to calculate a correlation slope $A . *,+, \#$ statistically significant difference $(p<0.05)$ between both patients' groups, non-lesional epilepsy and controls resp., lesional epilepsy and controls resp. MD - mean diffusivity, T2 - T2 relaxation times, Cho - choline containing compounds, $\mathrm{Cr}$ - total creatine, NAA - N-acetylaspartate $+\mathrm{N}$-acetylaspartylglutamate, $\mathrm{P}-$ positive correlation, $\mathrm{N}$ - negative correlation, $\varnothing-$ no correlation.

\section{Neuropathology}

Tissue specimens taken during resective surgery were fixed in a $10 \%$ neutral buffered formalin and embedded in paraffin. Routine hematoxylin-eosin (HE) and cresyl violet staining were performed. A significant loss of pyramidal neurons in both the CA1 and CA4 hippocampal sectors accompanied by gliosis was interpreted as HS. To assess the degree of gliosis in both the hippocampus and the resected neocortex, standard immunoperoxidase staining was performed using antibodies against the glial fibrillary acidic protein (clone 6F2, DakoCytomation).

Palmini's classification (Palmini et al. 2004) was used for evaluating malformations of cortical development.

\section{Results}

Electro-clinical, radiological, correlation and histopathological features for each patient are listed in Table 1. Correlations of metabolic data with MD and T2 were calculated for both the control and patient groups. In all groups $\mathrm{MD}$ and $\mathrm{T} 2$ (MD-T2) revealed positive correlation. MD, T2, Cho and metabolic ratios $\mathrm{Cr} / \mathrm{NAA}$, Cho/Cr, Cho/NAA gradually decreased (Figs 1, 2) and NAA gradually increased in the anteroposterior direction of the hippocampus in all subjects. The slopes and correlation coefficients are listed in Table 2.

\section{Correlation findings in the control group}

Positive correlation was found between T2 (or MD) and following metabolites (or metabolite ratios): Cho, Cr/NAA, Cho/Cr, Cho/NAA. NAA correlates with T2 or MD (NAA-T2, NAA-MD resp.) negatively and no correlation was found between $\mathrm{Cr}$ and T2 or MD (Table 2).

Based on the control group results, control intervals of each parameter were assessed and used for the subsequent semiautomatic evaluation (Figs 1,2).

\section{Correlation in non-lesional TLE patients}

Automatic evaluation based on control data revealed abnormal metabolic values in at least one $\mathrm{HC}$ in all patients with non-lesional epilepsy. In two cases, the abnormal values were found also in the contralateral HC and they were thus classified as a bilateral pathology with one side predominance (Table 1). In the remaining cases, the contralateral hippocampal findings corresponded to the healthy controls. The semiautomatic evaluation based on control data lateralized all the cases in accordance with the electro-clinical results. 

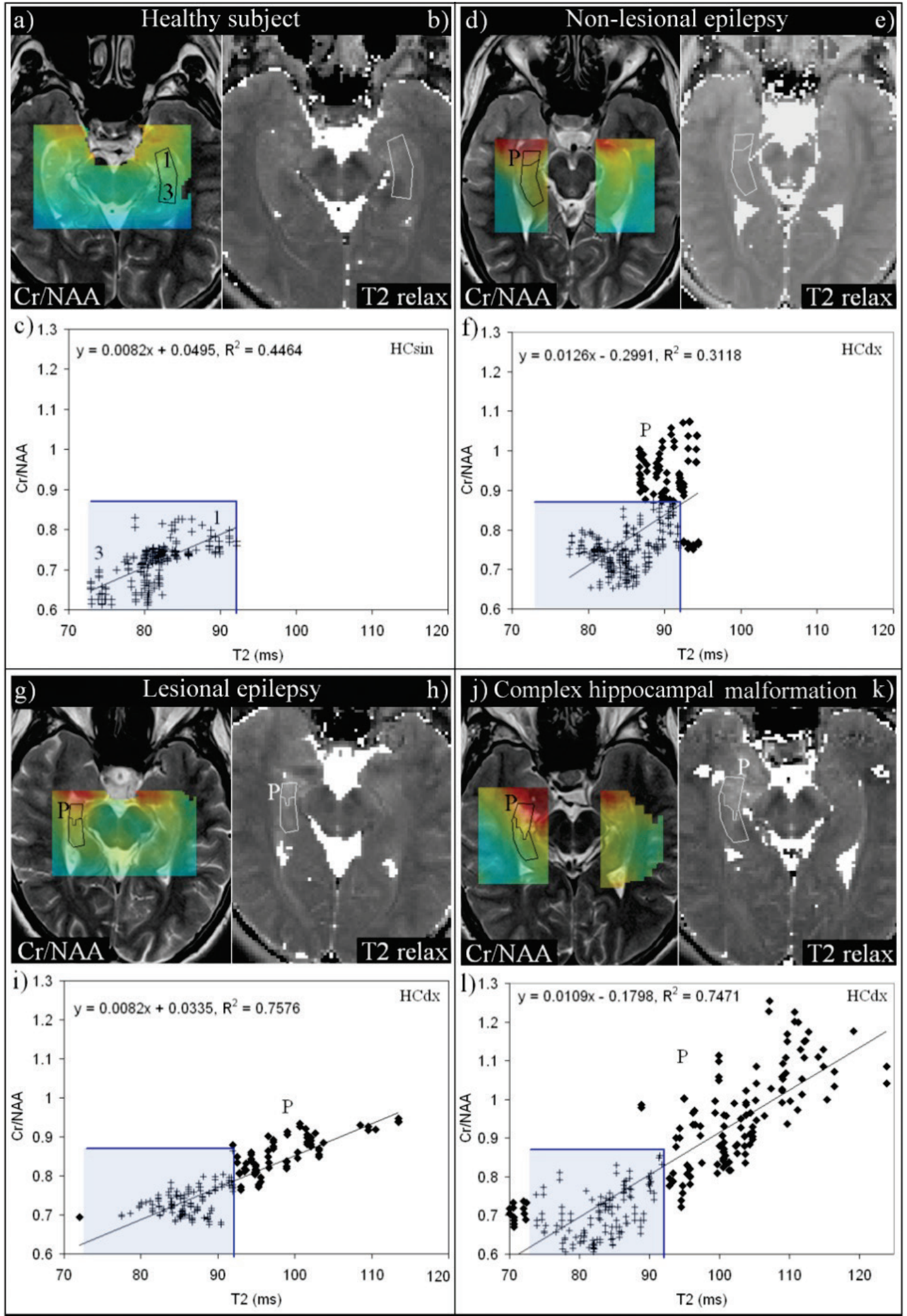

Fig. 1. The Cr/NAA-T2 correlations in hippocampi in a 24 year-old healthy control (a-c); in a 19 year-old female patient with non-lesional MTLE (d-f); in a 20 year-old male patient with right MTLE with HS (g-i); and in a 27 year-old male patient with right MTLE with complex malformation of the hippocampus $(\mathbf{j}-\mathbf{I}) . \mathbf{a}, \mathbf{d}, \mathbf{g}, \mathbf{j}$ : $\mathrm{Cr} / \mathrm{NAA}$ maps positioned on T2 weighted images with the maximal value of the Cramer-Rao bound of total $\mathrm{Cr} / \mathrm{NAA}$ ratios set to $15 \% ; \mathbf{b}, \mathbf{e}, \mathbf{h}, \mathbf{k}$ : T2 relaxation maps; $\mathbf{c}, \mathbf{f}, \mathbf{i}, \mathbf{l}$ : correlation plots; each cross represents an ordered pair of $\mathrm{Cr} / \mathrm{NAA}-\mathrm{T} 2$ values corresponding to one pixel in the analyzed area. The borders of controls are visible in correlation plots as blue lines. All the values outside the control interval visible as diamonds correspond to tissue abnormalities. According to semiautomatic quantitative evaluation, the anterior portion of the right hippocampus in patients showed an abnormal finding whereas the left hippocampus in a healthy control has normal finding. $\mathrm{Cr}$ - total creatine, NAA - N-acetyl aspartate + $\mathrm{N}$-acetylaspartylglutamate, $\mathrm{T} 2$ relax $-\mathrm{T} 2$ relaxation map, $\mathrm{HCdx}$ - right hippocampus, $\mathrm{HCsin}$ - left hippocampus, $\mathrm{P}$ - pathologic tissue. 

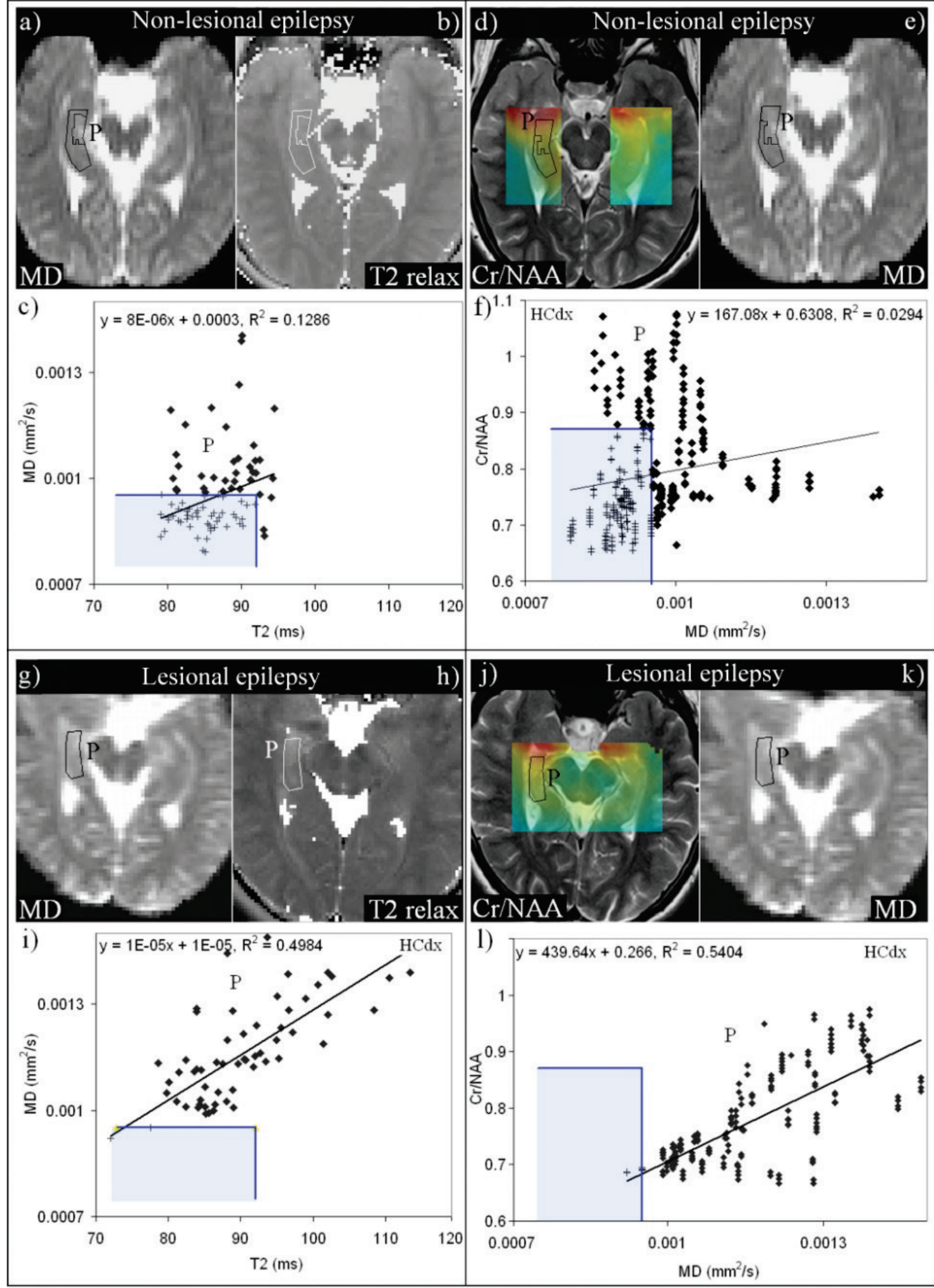

Fig. 2. The MD-T2 and $\mathrm{Cr} / \mathrm{NAA}-\mathrm{MD}$ correlations in the hippocampi in a 19 year-old female patient with right MTLE without HS (a-f); 20 year-old male patient with right MTLE with HS (g-l). $\mathbf{a}, \mathbf{e}, \mathbf{g}, \mathbf{k}:$ MD maps; $\mathbf{b}, \mathbf{h}$ : T2 relaxation maps; $\mathbf{d}, \mathbf{j}$ : $\mathrm{Cr} / \mathrm{NAA}$ maps positioned on T2 weighted images with the maximal value of the Cramer-Rao bound of total $\mathrm{Cr} / \mathrm{NAA}$ concentrations set to $15 \%$; $\mathbf{c}, \mathbf{f}, \mathbf{i}, \mathbf{l}$ : correlation plots in different scaling. Each cross represents an ordered pair of MD-T2 or Cr/NAA-MD values corresponding to one pixel in the analyzed area. The borders of controls data are visible in correlation plots as blue lines. All the values outside the control interval visible as diamonds correspond to tissue abnormalities. According to semiautomatic quantitative evaluation, the anterior portion of the right hippocampus in the patient without HS and entire $\mathrm{HC}$ in the patient with HS showed an abnormal finding. $\mathrm{Cr}-$ total creatine, NAA $\mathrm{N}$-acetyl aspartate $+\mathrm{N}$-acetylaspartylglutamate, $\mathrm{MD}$ - mean diffusivity, $\mathrm{T} 2$ relax $-\mathrm{T} 2$ relaxation map, $\mathrm{HCdx}$ - right hippocampus, $\mathrm{HCsin}$ - left hippocampus, $\mathrm{P}$ - pathologic tissue. 
Correlations in the $\mathrm{HC}$ with abnormal metabolic values are listed in Table 2 . In contrast to controls, $\mathrm{Cr}$ and T2 (Cr-T2) and $\mathrm{Cr}$ and MD (Cr-MD) correlated positively. The other correlations were similar to those in controls. The slopes of Cr/NAA-T2 and Cr-T2 dependence were significantly higher in patients than in the controls $(p<0.05)$. The difference is caused by substantial metabolic changes in patients, as the T2 and MD values are within the control intervals. The examples of correlations between $\mathrm{Cr} / \mathrm{NAA}$ and $\mathrm{T} 2$ (Cr/NAA-T2) are shown in Figure 1f.

\section{MR findings in lesional TLE patients}

Electro-clinical diagnosis (Table 1) and MRS-T2 evaluation lateralized EZ identically in all unilateral cases and the predominance in all bilateral cases. The results were confirmed by histopathology. The results of MRSMD evaluation corresponded to electro-clinical diagnosis in all unilateral cases and the predominance in five out of the seven bilateral cases.

Correlation patterns found in the $\mathrm{HC}$ with abnormal values are listed in Table 2 . In contrast to controls and patients with non-lesional TLE, Cho/Cr with T2 (or MD) did not significantly correlate. Other correlation patterns were similar to the non-lesional TLE patient group, however they exhibited stronger linear dependence (Table 2). The slopes of the Cr/NAA-T2 and $\mathrm{Cr}-\mathrm{T} 2$ correlations differed significantly from the controls $(\mathrm{p}<0.05)$. Moreover, Cr-T2(MD) and Cho-T2 slopes differed significantly from the non-lesional patients. The examples of $\mathrm{Cr} / \mathrm{NAA}-\mathrm{T} 2$ correlations are shown in Figures 1i, 11. Although Cr/NAA-T2 correlations exhibited trends of slopes smaller than in non-lesional patients, they did not reach statistical difference $(p=0.06)$.

All patients with lesional TLE exhibited increased T2 values in HC compared to healthy controls (11 patients unilaterally and 1 patient bilaterally). T2 values between $100 \mathrm{~ms}$ and $120 \mathrm{~ms}$ were found in both HS and hippocampal non-specific gliosis (histopathologically confirmed). Other lesions (ganglioglioma, complex hippocampal malformation associated with focal cortical dysplasia) exhibited even higher T2 (>120 ms).

\section{Discussion}

This work tested a new technique for studying mutual relationships between MR parameters based on a pixel-by-pixel image evaluation in groups of controls and patients with TLE. It proved that the relationships between metabolic and structural processes in pathologic hippocampus can be studied noninvasively. It brings a new general view of the pathology in an individual patient, which can be used in the assessment of hippocampal involvement or EZ lateralization. Although EZ lateralization based on correlations between the metabolic and T2 relaxation data agreed with electroclinical diagnosis in all tested cases, data processing is substantially complicated by measurement artifacts discussed below.

Mutual relationships between studied parameters in controls revealed gradual changes in individual parameters in the anteroposterior direction of the hippocampus and provide information about natural spatial distribution of these parameters across the hippocampus. The MD-T2 correlation (although weaker than in patients) reflects different morphology and showed increased extra- and intra-cellular space in the anterior portion compared to the posterior portion of the healthy hippocampus. The higher $\mathrm{T} 2$ relaxation times in the anterior $\mathrm{HC}$ portion are in accord with the previously reported studies (von Oertzen et al. 2002, Briellmann et al. 2004, Bartlett et al. 2007). We obtained significantly lower absolute values than the previous studies did. Nevertheless, von Oertzen himself mentioned that their method (dual-echo TSE sequence) resulted in higher T2 values than those measured with conventional relaxometry. We should also note that MD and T2 do not represent completely independent parameters as the diffusion measurements are weighted by relaxations and vice versa.

Different metabolic concentrations found in anteroposterior direction are also in agreement with previously reported studies performed at $1.5 \mathrm{~T}$ (Chu et al. 2000, Vermathen et al. 2000). Unfortunately, we cannot distinguish the contribution of chemical shift artifact (CSA) and hippocampal morphology (i.e. tissue changes in anteroposterior direction) to the correlations found in healthy controls. Although CSA may be negligible at $1.5 \mathrm{~T}$, it represents a significant contribution to correlations at $3 \mathrm{~T}$. Chu proposed a method for EZ lateralization using spectroscopy and tissue segmentation based on $\mathrm{T} 1$ values at $1.5 \mathrm{~T}$; however, we did not measure T1 maps and we found out that T2 maps were not suitable at $3 \mathrm{~T}$ due to substantial $\mathrm{T} 2$ changes in the lesions. Nevertheless, although CSA represents a possible systematic error, both control and patients' data are affected in the same direction. Therefore, in mutual 
comparisons, CSA has no relevant effect on the final results.

The pixel-by-pixel image analysis evaluates all the measured parameters in a complex way. Highlighted pixels with MR parameters out of the control interval clearly determine the pathology extent which is convenient for routine discrimination of the hippocampal abnormality. However, it seems that analysis of the correlation patterns and their slopes provides more detailed information about the significance of metabolic and structural changes in the pathologic tissue. Higher slopes in case of the positive correlations indicate severe metabolic abnormalities, while lower slopes severe structural changes. The changes of the slopes describe the tissue state and may assist in better presurgical planning especially in patients with negative MRI findings. Our findings are in accordance with previously reported results at 1.5T (Namer et al. 1999, Pereira et al. 2006) showing that the combination of different methods (i.e. MRI, 1H-MRS, T2 relaxometry and diffusometry) provides clinically relevant information additional to standard clinical assessment.

All measured correlations showed higher slopes in non-lesional TLE than in lesional epilepsy (Table 2), although not all differences are significant. Only Cr-T2 correlation slope of linear regression differs significantly between all subject groups. It indicates that reactive astrogliosis (represented by increased $\mathrm{Cr}$ ) and an increase of glial cell volume (increase of T2 values) are key factors in a hippocampal state characterization.

The correlation findings in non-lesional patients demonstrate no changes in the extra- and intra-cellular space (unchanged T2 and MD) but show neuronal dysfunction (decreased NAA) and reactive astrogliosis in the anterior portion of hippocampus. Biological barriers reducing ability of water diffusion therefore work properly and the tissue architecture is normally organized, without increased amount of glial cells (they have larger proportion of intracellular freely moving water - Pereira et al. 2006). These statements are in accordance with negative MRI findings in all these patients and with histopathological results in two patients who underwent surgery.

Patients with lesional TLE showed distinctive extra- and intra-cellular changes in the anterior portion of $\mathrm{HC}$ together with mild metabolic changes resulting in small slopes in positive linear correlations and high in negative ones. Therefore, distinctive neuronal loss or dysfunction and reactive astrogliosis are accompanied by characteristic architectural disorganization of the damaged tissue. Strong MD-T2 correlation further indicates that increased water mobility reflecting disrupt biological barriers and increased intracellular water movement resulting from increased amount of glial cells are associated processes. These findings were in accordance with histopathological results which showed hippocampal sclerosis (gliosis) or hippocampal nonspecific gliosis in the examined tissues. However, as both $\mathrm{HS}$ and $\mathrm{HC}$ non-specific gliosis exhibit increased $\mathrm{T} 2$, these two pathologies cannot be distinguished by correlation methods.

Linear regression fitted well correlations in controls and in patients with lesional epilepsy. In contrast, positive correlations in non-lesional patients seem to be non-linear or contain two components (Fig. 1f). We believe that it is a consequence of an inclusion of both healthy and pathological tissue into the examined region. Both tissues have normal architecture (negative MRI, normal MD and T2), but different metabolism represented by increased $\mathrm{Cr}$ and decreased NAA in patients.

The proposed correlation method may be sensitive to age as the pathologic tissue is automatically detected according to data from the control group. MR parameters change significantly in small children during brain maturation (Kreis et al. 1993, Pouwels et al. 1999) and in elderly people due to demyelination and degradation processes. In these cases, age matched subjects should be used in control database to avoid underestimation or overestimation of the pathology extent. Nevertheless, MR parameters in adolescents and adults are supposed to be stable and the use of one control group is sufficient.

The correlations of metabolic values with relaxation times were found to be the best markers for assessment of hippocampal involvement. The semiautomatic evaluation of correlations of other parameters with MD failed due to distortions in diffusion images leading to the increased MD values in the vicinity of the brain stem. Increased image distortions in the image plane containing both temporal and facial regions are caused by an inherent sensitivity of echo planar imaging sequences to inhomogeneities of the static magnetic field. It could be corrected during images postprocessing using an additional B0 map.

In our study, the metabolite concentrations were corrected neither for relaxation times nor for cerebrospinal fluid volume because it was unnecessary 
for the proposed method and the exact absolute concentrations were not the stated aim of the study. Nevertheless, lack of these corrections has no effect on metabolic ratios.

Single voxel spectroscopy over the larger volume of the hippocampus may be sufficient for basic lateralization (Kuzniecky 1999, Hajek et al. 2008). However, the existence of correlation requires comparison of the patients' data and controls in the same portion of HC (Vermathen et al. 2000), as the values of metabolite concentration, T2 and MD gradually change in the anteroposterior direction. Averaging of the values over the whole hippocampus leads to a substantial data dispersion related to physiological properties of the tissue.

The CORIMA program reciprocally highlights pixels corresponding to control data using thresholds determined as the mean values \pm standard deviations. Therefore even in the healthy region may occur values exceeding the control interval of a corresponding MR parameter. This can be observed in Figures 1i, 11 (the pixels with very low T2 parameters). These values did not exceed the control interval by more than $5 \%$ in T2 values and metabolic concentrations; however, MD values in the vicinity of the brain stem may exceed the MAX value by more than $20 \%$. Inclusion or exclusion of high MD values in the vicinity of the brain stem from the volunteers' statistics strongly affects the control interval and therefore may underestimate or overestimate the pathology extent in patients (Fig. 2). The analysis alongside the diffusion distortions may lead to the failure of the MRS-MD assessment. Therefore, this method should be used only as semiautomatic with the supervision of an operator.

In conclusion, description of an extent and character of hippocampal pathology in patients with TLE can be substantially improved by a detailed analysis of multiple MR parameters. Evaluation of correlation patterns of the quantitative parameters along the anteroposterior direction of the hippocampus represents a suitable approach. The slopes of $\mathrm{Cr} / \mathrm{NAA}-\mathrm{T} 2$ and $\mathrm{Cr}-\mathrm{T} 2$ correlations differed significantly between patients and controls and are the best biomarkers of the epileptogenic foci. The proposed method based on a pixel-by-pixel evaluation of MR images may supplement appropriately a standard clinical protocol. However, the automated processing should be carefully monitored in order to avoid errors caused by possible MR artifacts.

\section{Conflict of Interest}

There is no conflict of interest.

\section{Acknowledgements}

The study was supported by the following grant projects: IGA MZCR NS 9915-4/2008, MH CZ - DRO („Institute for Clinical and Experimental Medicine - IKEM, IN 00023001“), MH CZ - DRO („University Hospital Motol, Prague, Czech Republic 00064203“).

\section{References}

BARTLETT PA, SYMMS MR, FREE SL, DUNCAN JS: T2 relaxometry of the hippocampus at 3T. AJNR Am $J$ Neuroradiol 28: 1095-1098, 2007.

BONILHA L, EDWARDS JC, KINSMAN SL, MORGAN PS, FRIDRIKSSON J, RORDEN CH, RUMBOLDT Z, ROBERTS DR, ECKERT MA, HALFORD JJ: Extrahippocampal gray matter loss and hippocampal deafferentation in patients with temporal lobe epilepsy. Epilepsia 51: 519-528, 2010.

BRIELLMANN RS, SYNGENIOTIS A, FLEMING S, KALNINS RM, ABBOTT DF, JACKSON GD: Increased anterior temporal lobe $\mathrm{T} 2$ times in cases of hippocampal sclerosis: a multi-echo T2 relaxometry study at $3 \mathrm{~T}$. AJNR Am J Neuroradiol 25: 389-394, 2004.

CHU WJ, KUZNIECKY RI, HUGG JW, ABOU-KHALIL B, GILLIAM F, FAUGHT E, HETHERINGTON HP: Statistically driven identification of focal metabolic abnormalities in temporal lobe epilepsy with corrections for tissue heterogeneity using 1H spectroscopic imaging. Magn Reson Med 43: 359-367, 2000.

CONCHA L, BEAULIEU C, COLLINS DL, GROSS DW: White-matter diffusion abnormalities in temporal-lobe epilepsy with and without mesial temporal sclerosis. J Neurol Neurosurg Psychiatry 80: 312-319, 2009.

DUZEL E, KAUFMANN J, GUDERIAN S, SZENTKUTI A, SCHOTT B, BODAMMER N, HOPF M, KANOWSKI M, TEMPELMANN C, HEINZE HJ: Measures of hippocampal volumes, diffusion and 1H MRS metabolic abnormalities in temporal lobe epilepsy provide partially complementary information. Eur J Neurol 11: 195-205, 2004. 
ENGEL J JR: Introduction to temporal lobe epilepsy. Epilepsy Res 26: 141-150, 1996.

FSL [computer program]. Available via http://www.fmrib.ox.ac.uk/fsl/

HAJEK M, DEZORTOVA M, KRSEK P: 1H MR spectroscopy in epilepsy. Eur J Radiol 67: 258-267, 2008.

HERYNEK V, WAGNEROVA D, HEJLOVA I, DEZORTOVA M, HAJEK M: Changes in the brain during long-term follow-up after liver transplantation. J Magn Reson Imaging 35: 1332-1337, 2012.

JACK CR: Hippocampal T2 relaxometry in epilepsy: past, present, and future. AJNR Am J Neuroradiol 17: 1811-1914, 1996.

JIRU F, SKOCH A, KLOSE U, GRODD W, HAJEK M: Error images for spectroscopic imaging by LCModel using Cramer-Rao bounds. Magn Reson Mater Phy 19: 1-14, 2006.

JIRU F, SKOCH A, WAGNEROVA D, DEZORTOVA M, HAJEK M: jSIPRO - analysis tool for magnetic resonance spectroscopic imaging. Comput Methods Programs Biomed 112: 173-188, 2013.

KANTARCI K, SHIN C, BRITTON JW, SO EL, CASCINO GD, JACK CR JR: Comparative diagnostic utility of 1H MRS and DWI in evaluation of temporal lobe epilepsy. Neurology 58: 1745-1753, 2002.

KELLER SS, ROBERTS N: Voxel-based morphometry of temporal lobe epilepsy: an introduction and review of the literature. Epilepsia 49: 741-757, 2008.

KNAKE S, SALAT DH, HALGREN E, HALKO MA, GREVE DN, GRANT PE: Changes in the white matter microstructure in patients with TLE and hippocampal sclerosis. Epileptic Disord 11: 244-250, 2009.

KREIS R, ERNST T, ROSS BD: Development of the human brain: in vivo quantification of metabolite and water content with proton magnetic resonance spectroscopy. Magn Reson Med 30: 424-437, 1993.

KUZNIECKY R: Magnetic resonance spectroscopy in focal epilepsy: 31P and $1 \mathrm{H}$ spectroscopy. Rev Neurol 155: 495-498, 1999.

KUZNIECKY R, PALMER CH, HUGG J, MARTIN R, SAWRIE S, MORAWETZ R, FAUGHT E, KNOWLTON R: Magnetic resonance spectroscopic imaging in temporal lobe epilepsy: Neuronal dysfunction or cell loss? Arch Neurol 58: 2048-2053, 2001.

MARGERISON JH, CORSELLIS JAN: Epilepsy and the temporal lobe. A clinical, electroencephalographic and neuropathological study of the brain in epilepsy, with particular reference to the temporal lobes. Brain $\mathbf{8 9}$ : 499-530, 1966.

NAMER IJ, BOLO NR, SELLAL F, NGUYEN VH, NEDELEC JF, HIRSCH E, MARESCAUX C: Combined measurements of hippocampal N-acetyl-aspartate and $\mathrm{T} 2$ relaxation times in the evaluation of mesial temporal lobe epilepsy: correlation with clinical severity and memory performances. Epilepsia 40: 1424-1432, 1999.

PALMINI A, NAJM I, AVANZINI G, BABB T, GUERRINI R, FOLDVARY-SCHAEFER N, JACKSON G, LUDERS HO, PRAYSON R, SPREAFICO R, VINTERS HV: Terminology and classification of the cortical dysplasias. Neurology 62: S2-S8, 2004.

PEREIRA PMG, OLIVEIRA E, ROSADO P: Apparent diffusion coefficient mapping of the hippocampus and the amygdala in pharmacoresistant temporal lobe epilepsy. AJNR Am J Neuroradiol 27: 671-683, 2006.

POUWELS PJW, BROCKMANN K, KRUSE B, WILKEN B, WICK M, HANEFELD F, FRAHM J: Regional age dependence of human brain metabolites from infancy to adulthood as detected by quantitative localized proton MRS. Pediatr Res 46: 474-485, 1999.

PROVENCHER SW: Estimation of metabolite concentrations from localized in vivo proton NMR spectra. Magn Reson Med 30: 672-679, 1993.

VERMATHEN P, LAXER KD, MATSON GB, WEINER MW: Hippocampal structures: anteroposterior $\mathrm{N}$-acetylaspartate differences in patients with epilepsy and control subjects as shown with proton MR spectroscopic imaging. Radiology 214: 403-410, 2000.

VON OERTZEN J, URBACH H, BLUMCKE I, REUBER M, TRABER F, PEVELING T, MENZEL C, ELGER CE: Time-efficient T2 relaxometry of the entire hippocampus is feasible in temporal lobe epilepsy. Neurology $\mathbf{5 8}$ : 257-264, 2002.

WAGNEROVA D, JIRU F, DEZORTOVA M, VARGOVA L, SYKOVA E, HAJEK M: The correlation between 1H MRS choline concentrations and MR diffusion trace values in human brain tumours. Magn Reson Mater Phy 22: 19-31, 2009. 